\title{
Comparaison des activités alimentaires et méryciques d'ovins et de caprins recevant de la paille d'orge traitée ou non à la soude
}

\author{
C. Masson, D. Kirilov, F. Faurie et J.L. Tisserand \\ ENSSAA, laboratoire de recherches INRA de la chaire de zootechnie, 26, bd, Docteur-Petitjean, \\ 21000 Dijon, France
}

(recu le 29 septembre 1988, accepté le 24 mai 1989)

\begin{abstract}
Résumé - Les activités alimentaires et méryciques de 4 boucs Alpin et de 4 béliers lle-de-France, castrés, âgés de 2 ans et pesant en moyenne 50 et $45 \mathrm{~kg}$, ont été enregistrées successivement pour 2 fourrages : une paille d'orge non traitée, puis traitée à la soude $(4 \%)$, les 2 supplémentées avec $120 \mathrm{~g}$ de tourteau de soja par $\mathrm{kg}$ de paille ingérée. La quantité totale de matière sèche ingérée par kg Po.75 a été plus élevée chez les caprins que chez les ovins; elle est supérieure de 25 et $11 \%$ respectivement pour la paille témoin et celle traitée à la soude. Ce résultat est obtenu par une consommation diurne plus importante chez les caprins. Le traitement de la paille par la soude augmente les quantités ingérées de 30 et $47 \%$ respectivement chez les caprins et chez les ovins. La durée d'ingestion n'a pas varié selon les espèces. Elle est, en moyenne, de 262 min chez les caprins et de 257 min chez les ovins. La durée unitaire d'ingestion est toujours inférieure chez les caprins, qui font davantage de repas dans la journée, mais des repas plus courts que les ovins. La distribution de paille sodée augmente plus la vitesse d'ingestion chez les caprins $(+58 \%)$ que chez les ovins $(+30 \%)$. Les caprins mastiquent plus longtemps que les ovins avec le régime paille témoin et c'est l'inverse avec la paille sodée. La distribution de paille sodée augmente plus la vitesse de mastication chez les caprins que chez les ovins (+ $58 \%$ et $+18 \%$ respectivement). La consommation d'eau exprimée en $\mathrm{ml} / \mathrm{g} \mathrm{MSI}$ est inférieure chez les caprins pour les 2 régimes (en moyenne 5,3 et $6,2 \mathrm{ml} / \mathrm{g}$ MSI respectivement chez les caprins et chez les ovins). Par rapport aux ovins, avec la paille témoin, les caprins ruminent plus pendant la nuit tandis qu'avec la paille traitée à la soude, ils ruminent moins. La durée unitaire de rumination est inférieure, dans l'ensemble, chez les caprins et quelle que soit l'espèce animale, elle est plus élevée avec le régime paille témoin $(9,8 \mathrm{~min} / \mathrm{g} \mathrm{MS} / \mathrm{kg} \mathrm{P0,75})$ qu'avec le régime paille sodée $\left(7,1 \mathrm{~min} / \mathrm{g} \mathrm{MS} / \mathrm{kg} \mathrm{P}^{0.75}\right)$.
\end{abstract}

Ingestion - rumination - ovins - caprins - paille

Summary - Feeding behaviour and rumination in sheep and goats fed with sodium hydroxide treated or untreated barley straw. Feeding and rumination behaviour were studied in four Alpine wether goats and four lle de France wether sheep of similar age (2 yr). Two diets were used successively : an untreated and a sodium hydroxide (4\%) treated barley straw supplemented with $120 \mathrm{~g}$ of soya bean meal by $\mathrm{kg}$ of ingested straw. Goats ingested more dry matter (g DM/kg W 0.75 ) than sheep; the increase was $25 \%$ and $11 \%$, respectively for untreated and treated straw. This was due to the higher diurnal intake in goats. The sodium hydroxide treatment of straw increased voluntary intake by $30 \%$ and $47 \%$, respectively in goats and sheep. The time spent eating did not vary between the two animal species (262 $\mathrm{min}$ and $257 \mathrm{~min}$ in goats and sheep respectively). The unitary eating time was always lower in goats which ingested more often smaller meals during the day than sheep. With treated straw, the feeding rate increased more in goats $(+58 \%)$ than in sheep $(+30 \%)$. Goats masticated over a longer period of time than sheep with untreated straw and it was the contrary with treated straw. With treated straw, the mastication rate increased more in goats (+ $58 \%)$ than in sheep (+18\%). Water consumption in $\mathrm{ml} / \mathrm{g} I D M$ was lower in goats with the two diets 
(on average 5.3 and $6.2 \mathrm{ml} / \mathrm{g} I D M$, respectively in goats and sheep). The unitary ruminating time was lower in goats and whatever the animal species, it was more important with untreated straw $(9.8 \mathrm{mn} / \mathrm{g}$ $\left.D M / \mathrm{kg} \mathrm{W}^{0.75}\right)$ than with treated straw $\left(7.1 \mathrm{mn} / \mathrm{g} \mathrm{DM} / \mathrm{kg} \mathrm{W}^{0,75}\right)$.

voluntary intake - rumination - sheep - goats - straw

\section{INTRODUCTION}

Dans certaines conditions d'élevage (zones arides ou semi-arides), la digestion des fourrages pauvres apparaît plus efficace chez les caprins que chez les ovins (Elhag, 1976; Gihad, 1976; Brown \& Johnson, 1984). Cette différence résulterait d'un comportement de tri, permettant un choix alimentaire plus efficient chez les caprins (Devendra, 1981; Morand-Fehr, 1981). De plus, une rumination moins efficiente limiterait l'aptitude des ovins à utiliser des fourrages trop fibreux (Welch, 1982). Ainsi, une meilleure connaissance des activités alimentaires et méryciques pourrait-elle contribuer à expliquer certaines différences de digestion entre les espèces de ruminants (Dulphy et al., 1979; Dulphy \& Michalet-Doreau, 1983). A ce jour, peu d'études ont été réalisées simultanément chez les ovins et les caprins (Geoffroy, 1974; Alrahmoun, 1984; Focant, 1984; Focant et al., 1986; Dulphy et al., 1984; Dulphy \& Carle, 1986) : c'est pourquoi nous avons enregistré le comportement alimentaire et mérycique de boucs et de béliers castrés, alimentés avec de la paille d'orge traitée ou non à la soude.

\section{MATÉRIEL ET MÉTHODES}

\section{Animaux}

Quatre boucs castrés de race Alpine et 4 béliers de race lle-de-France élevés dans les mèmes conditions, âgés de 2 ans et demi et pesant en moyenne respectivement 50 et $45 \mathrm{~kg}$ ont été utilisés dans cet essai. Ces poids, qui pourraient être considérés comme faibles pour des sujets adultes, résultent du fait que les animaux avaient été affectés depuis plusieurs mois à des expériences sur fourrages pauvres.

\section{Aliments}

Une paille d'orge a été traitée à la soude par la voie semi-sèche à l'aide d'une machine JFSP 2000 , utilisant 12 I de lessive de soude à $30 \%$ pour $100 \mathrm{~kg}$ de paille, ce qui représente un taux de soude incorporée de $4 \%$.

La composition chimique et la digestibilité ( $M O$ et $C B$ ) de la paille avant (paille témoin) et après traitement (paille sodée : PS) sont données dans le Tableau I.

Au cours de 2 périodes successives de 5 semaines chacune, ces animaux ont reçu de la paille d'orge traitée à la soude, puis la même paille non traitée, complémentées dans les 2 cas avec $120 \mathrm{~g}$ de tourteau de soja par $\mathrm{kg}$ de fourrage. Les pailles ont été distribuées ad libitum ( $10 \%$ refus minimum) en 2 repas égaux par jour ( $8 \mathrm{~h}$ et $16 \mathrm{~h} 30)$. Des pierres à lécher et de l'eau sont restées en permanence à la disposition des animaux.

\section{Mesures}

Après 3 semaines d'accoutumance, les quantités de paille et d'eau de boisson consommées ont été mesurées individuellement et quotidiennement (entre $8 \mathrm{~h} 30$ et $16 \mathrm{~h}$ et entre $16 \mathrm{~h} 30$ et $8 \mathrm{~h}$ ) durant 2 semaines. Le mouvement de mâchoire de chaque sujet a été enregistré durant 4 jours consécutifs à l'aide de vibrographes de Kienzle TFW $3 / 8$ décrits par Ruckebusch \& Bueno (1973) et modifiés par Bechet (1978). 
Tableau I. Composition chimique des aliments.

\begin{tabular}{|c|c|c|c|c|c|c|c|}
\hline Aliments & $\begin{array}{c}\text { Matière sèche } \\
\mathrm{g} / \mathrm{kg}\end{array}$ & $\begin{array}{c}\text { Cellulose brute } \\
\text { g/kg MS }\end{array}$ & $\begin{array}{c}\text { Matière azotée } \\
\text { g/kg MS }\end{array}$ & $\begin{array}{l}\text { Cendres } \\
\text { g/kg MS }\end{array}$ & $\begin{array}{l}\text { Lignine } \\
\text { GKg MS }\end{array}$ & $\begin{array}{l}\text { Dige } \\
\text { MO }\end{array}$ & $\begin{array}{c}\text { ilité \% } \\
C B\end{array}$ \\
\hline Paille d'orge & 93,9 & 45,9 & 3,9 & 5,0 & 7,1 & 46,7 & 56,0 \\
\hline $\begin{array}{l}\text { Paille d'orge } \\
\text { traitée à la } \\
\text { soude }\end{array}$ & 92,7 & 46,1 & 3,7 & 8,6 & 7,4 & 52,1 & 66,6 \\
\hline
\end{tabular}

Les durées totales et unitaires $(\mathrm{min} / \mathrm{g} \mathrm{MSI}$ paille/kg $\mathrm{P}^{0.75}$ ), le nombre et la durée moyenne des périodes d'ingestion et de rumination sont rapportées à la consommation de paille.

Les différents résultats sont présentés séparément pour la période située entre le repas du matin $(8$ h 30$)$ et celui du soir (16 h 30) appelé "jour" et le reste de la période de $24 \mathrm{~h}$, appelé "nuit", ainsi que pour l'ensemble du nycthémère. Une analyse de variance à 2 facteurs, régime et espèce, a été réalisé pour les paramètres concernant l'ingestion et la rumination sur $24 \mathrm{~h}$.

\section{RÉSULTATS}

\section{Ingestion}

La quantité totale de matière sèche ingérée par jour (en $\mathrm{g} / \mathrm{kg} \mathrm{P}^{0,75}$ ) est significativement plus élevée chez les caprins que chez les ovins $(+25 \%$ et $+11 \%$ respectivement pour la paille témoin et la paille sodée) [Tableau II]. Les caprins ingèrent autant le jour que la nuit, alors que les ovins ont une consommation diurne plus faible, en particulier avec la paille sodée. La durée d'ingestion est supérieure chez les caprins $(+12 \%)$, par rapport aux ovins, avec la paille témoin, mais l'inverse se produit avec la paille sodée $(-8 \%)$. Si le traitement de la paille ne modifie pas la durée d'ingestion chez les ovins, il la réduit sensiblement chez les caprins (-12\%).

Les caprins mangent plus vite que les ovins, $+15 \%$ et $+40 \%$ respectivement pour la paille témoin et la paille sodée; le traitement de la paille entraîne une augmentation de la vitesse d'ingestion chez les caprins $(+58 \%$ ) par rapport aux ovins (+ $90 \%$ ). Les caprins font des repas plus nombreux et plus courts que les ovins. Avec la paille sodée et quelle que soit l'espèce animale, le nombre de repas est augmenté et leur durée est diminuée.

Par tête ou par $\mathrm{kg}$ de poids métabolique, la consommation d'eau ne diffère pas statistiquement entre les ovins et les caprins, mais, par $\mathrm{g}$ de MSI, elle est inférieure chez les caprins pour la paille témoin comme pour la paille sodée (Tableau III). L'ingestion de cette dernière augmente la consommation d'eau de façon sensiblement égale chez les ovins et les caprins (+ 62 et $+70 \%$ respectivement). 


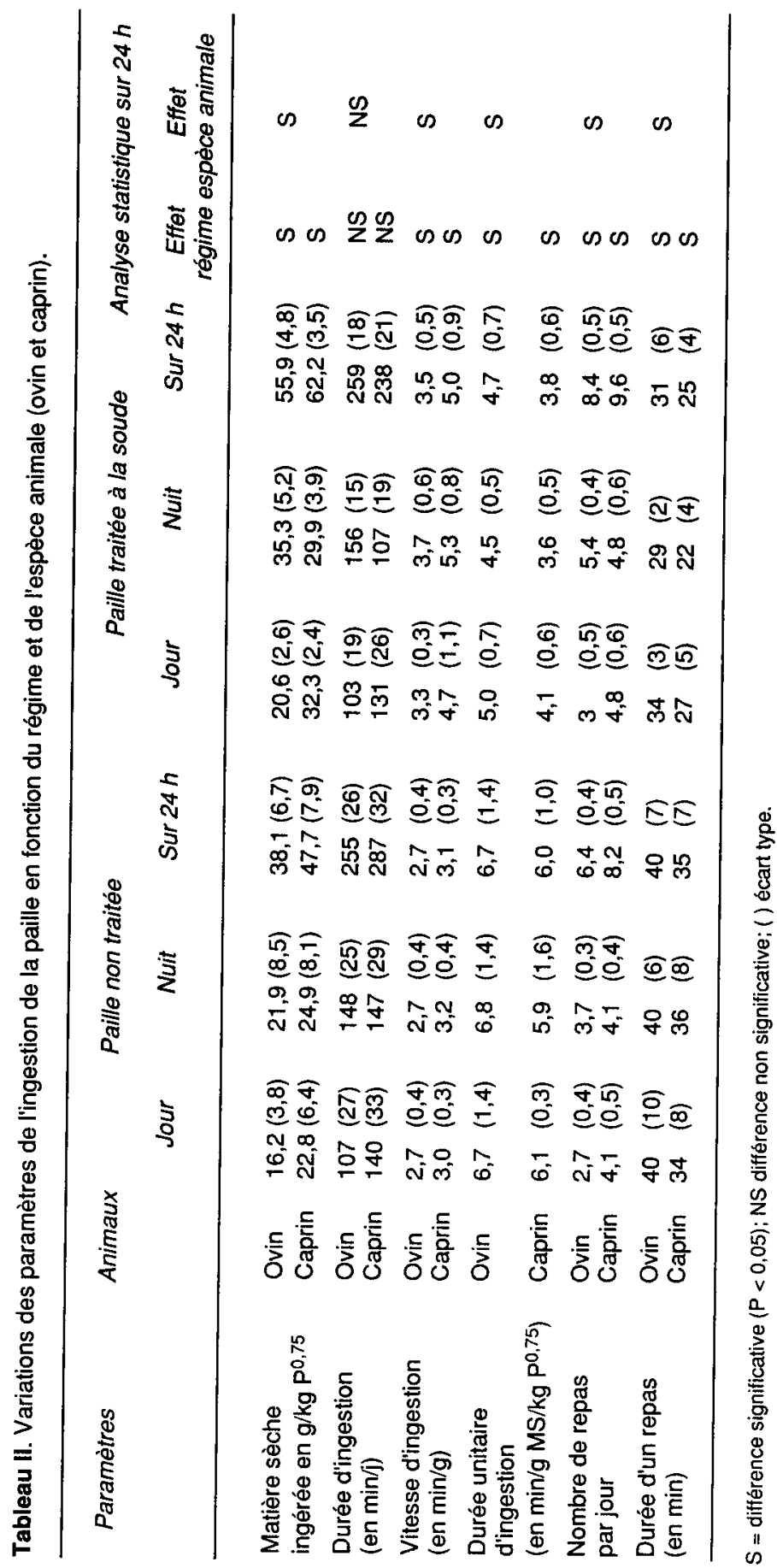




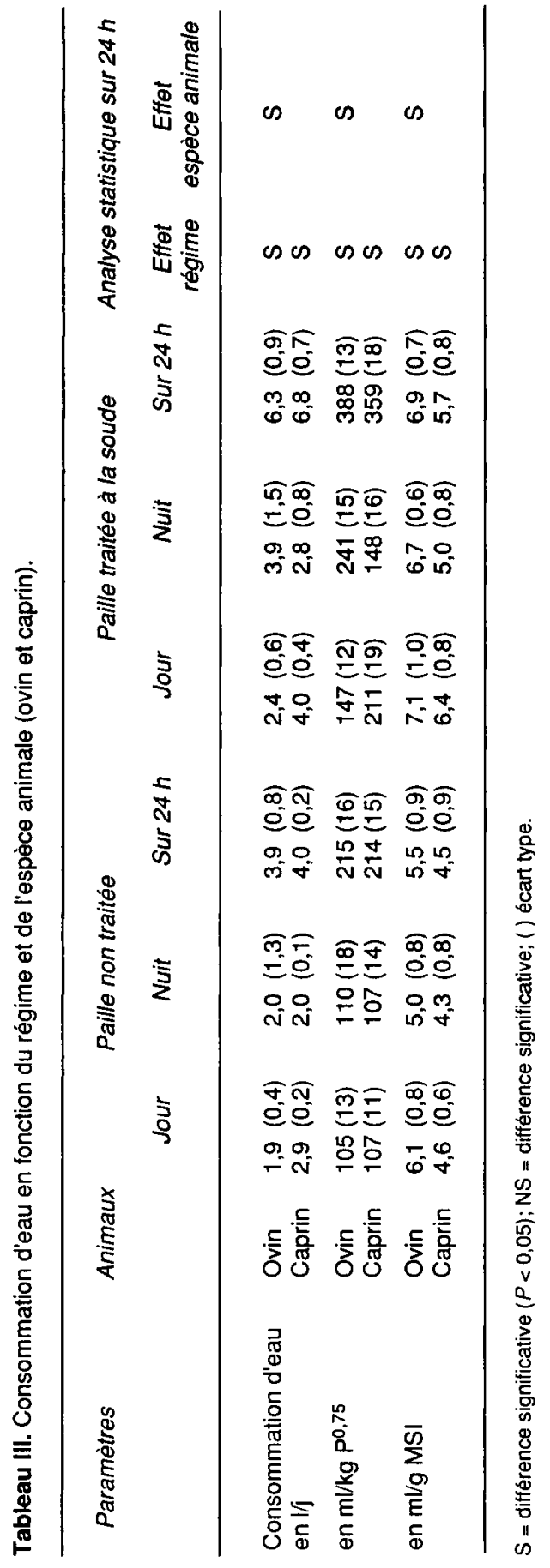




\section{Rumination}

La durée de rumination, exprimée en min par 24 h, est influencée aussi bien par l'espèce animale que par le régime (Tableau IV). Toutefois, les caprins et les ovins ne réagissent pas de la même façon vis-à-vis de l'aliment. Avec la paille témoin, les caprins ruminent plus $(+21 \%$ ) que les ovins mais, avec la paille sodée, ils ruminent moins $(-11 \%)$. Les caprins ruminent un peu plus rapidement que les ovins, $+11 \%$ et $+43 \%$ respectivement pour la paille témoin et la paille sodée.

Le traitement de la paille à la soude augmente plus la vitesse de rumination chez les caprins que chez les ovins, $+50 \%$ et $+16 \%$ respectivement, et il diminue plus la durée unitaire de rumination chez les caprins $(-35 \%)$ que chez les ovins $(-21 \%)$.

Le nombre de périodes de rumination ainsi que leur durée ne diffèrent pas statistiquement entre les 2 espèces.

\section{Mastication}

Lorsqu'ils consomment la paille témoin, les caprins mastiquent plus longtemps $(+17 \%)$ que les ovins (Tableau V), alors que, avec la paille sodée, leur temps de mastication est réduit $(-10 \%)$ par rapport aux ovins. Le traitement à la soude augmente la durée de mastication chez les ovins $(+11 \%)$ mais la diminue chez les caprins $(-15 \%)$. La vitesse de mastication est la même pour les ovins et les caprins lorsqu'ils consomment la paille témoin. La distribution de paille sodée augmente plus la vitesse de mastication chez les caprins que chez les ovins $(+58$ et $+18 \%$ respectivement).

\section{DISCUSSION ET CONCLUSION}

Les quantités de paille ingérées et les paramètres du comportement alimentaire et mérycique diffèrent donc entre les caprins et les ovins, ce qui confirme les observations faites par plusieurs auteurs avec des fourrages pauvres, comme le soulignent Brown \& Johnson (1984) dans leur revue bibliographique.

Pour les 2 régimes étudiés :

- les caprins mangent plus et boivent moins dans la mesure où la quantité d'eau consommée est rapportée à la matière sèche ingérée;

- par rapport aux ovins, les vitesses d'ingestion, de mastication et de rumination sont plus élevées chez les caprins, les durées unitaires d'ingestion, de mastication et de rumination étant alors logiquement plus faibles;

- les caprins font davantage de repas, mais moins longs, que les ovins;

- le traitement à la soude améliore plus le niveau d'ingestion de la paille chez les caprins ( $+46 \%$ contre $+30 \%$ pour les ovins).

Nos résultats sont donc en accord avec ceux rapportés par Masson et al. (1986). Les caprins sembleraient mieux s'adapter que les ovins aux fourrages pauvres ayant une teneur élevée en glucides pariétaux.

Par rapport à la paille traitée à la soude, la distribution de paille témoin montre que les caprins seraient plus aptes que les ovins à augmenter leur durée d'ingestion ( $+20 \%$ contre $-2 \%$ pour les ovins), tout en diminuant leur vitesse d'ingestion ( $-37 \%$ contre $-23 \%$ chez les ovins). Ils augmentent leur durée de mastication $(+17 \%)$ et leur durée de rumination $(+16 \%)$, alors que ces 2 valeurs diminuent chez les ovins $(-10 \%$ et $-15 \%$ respectivement). 


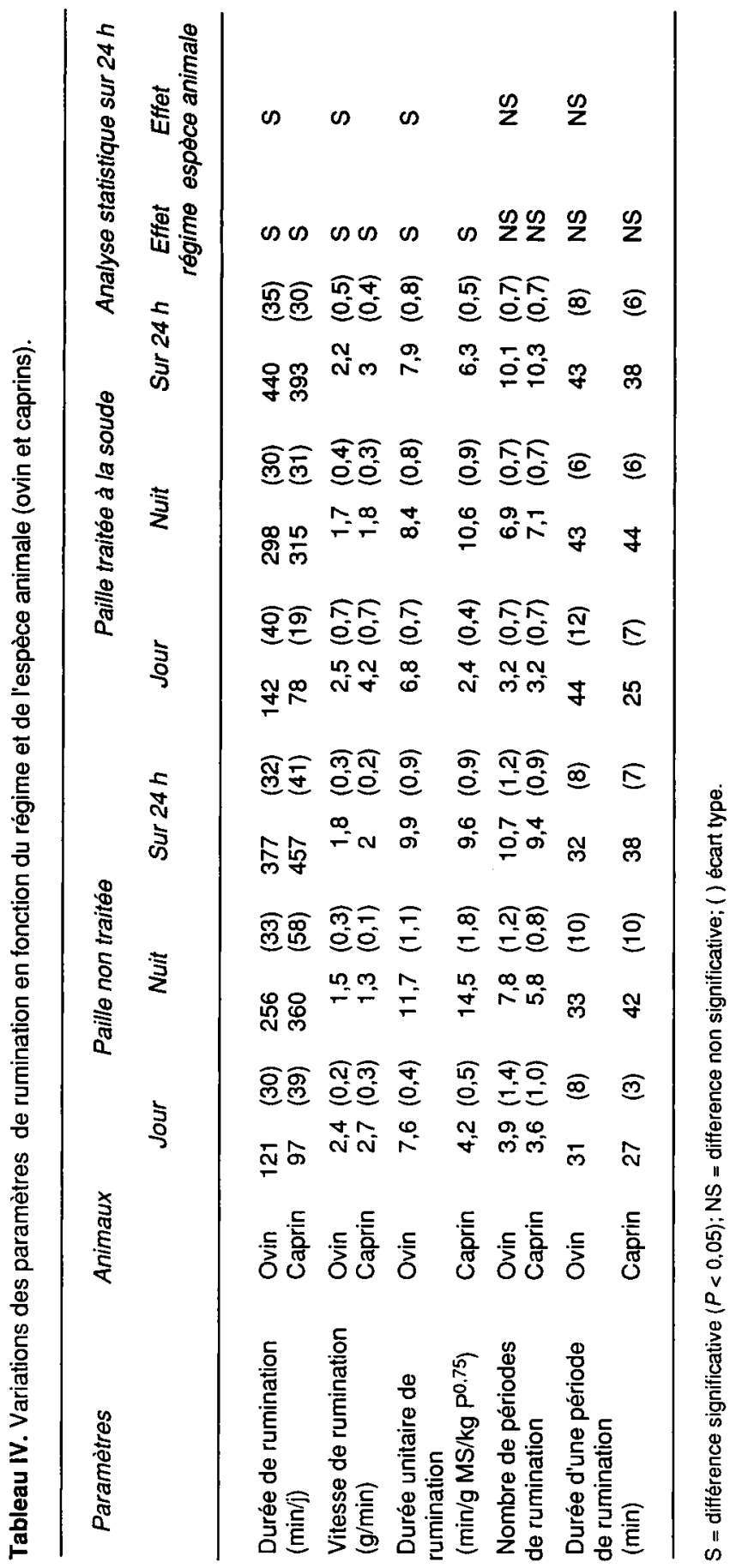




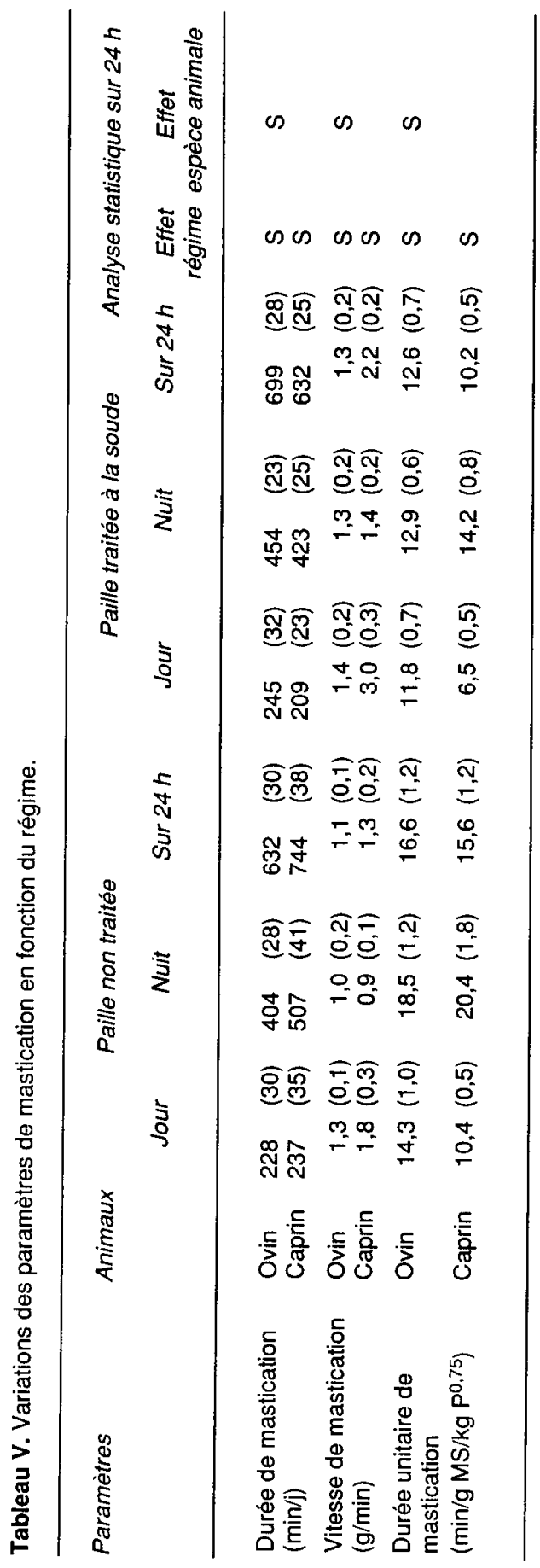


La dégradation in sacco de la lignocellulose (ADF Van Soest) des mêmes lots de paille, mesurée sur une période de $48 \mathrm{~h}$, chez des ovins nourris soit avec du foin, soit avec de la paille, indique que la paille témoin représente respectivement 62 et $81 \%$ de la paille sodée.

Cette étude tendrait à démontrer qu'il existe, en ce qui concerne le comportement alimentaire des caprins, un mécanisme d'adaptation plus efficace que chez les ovins et qui varie en raison inverse de la qualité des fourrages, comme le confirment les résultats de Focant (1984), lequel montre, avec les rations riches en concentré, que les caprins ruminent moins que les ovins.

Cela expliquerait, d'autre part, l'apparente contradiction entre nos résultats et ceux de Geoffroy (1974), lequel enregistre des durées unitaires d'ingestion plus faibles chez le mouton pour la consommation d'un bon foin et d'un ensilage distribué ad libitum.

D'autres études, avec une gamme plus large de régimes, en y associant l'étude de la composition de l'ingéré réel et des bols œsophagiens, la digestion microbienne dans le cæcum et la vitesse de transit, devraient nous permettre d'analyser plus finement les différences entre ces 2 espèces de petits ruminants.

\section{RÉFÉRENCES}

Alrahmoun W. (1984) Etude comparée de l'activité microbienne dans le rumen chez les caprins et les ovins. Thèse de doctorat d'État, Dijon

Bechet G. (1978) Enregistrement des activités alimentaires et méryciques des ovins au pâturage. Ann. Zootech. 27, 107-113

Brown L.E. \& Johnson W.L. (1984) Comparative intake and digestibility of forages and by- products by goats and sheep : a review. Intern. Goat Sheep Res. 3, 212-226

Devendra C. (1981) The utilization of forages from cassava pigeon pea, leucaena and groundnut by goats and sheep in Malaysia : In: Symposium International "Nutrition et Systèmes d'Alimentation de La Chèvre", vol. 1 (Morand-Fehr P., Bourbouze A. \& De Simiane M., eds). ITOVIC, INRA, Tours, pp. 338-345

Dulphy J.P., Remond B. \& Theriez M. (1979) Ingestive behaviour and related activities in ruminants. In : Proc. 5th Int. Symp. on Digestive Physiology and Metabolism in Ruminants. Clermont-Ferrand, 3-7 septembre (Ruckebusch Y. \& Thivend P., eds). MTP Press Ltd, Lancaster, pp. 103-122

Dulphy J.P. \& Michalet-Doreau B. (1983) Comportement alimentaire et mérycique d'ovins et de bovins recevant des fourrages verts. Ann. Zootech. 32, 465-474

Dulphy J.P., Michalet-Doreau B. \& Demarquilly C. (1984) Etude comparée des quantités ingérées et du comportement alimentaire et mérycique d'ovins et de bovins recevant des ensilages d'herbe réalisés selon différentes techniques. Ann. Zootech. 33, 291-320

Dulphy J.P. \& Carle B. (1986) Activités alimentaires et méryciques comparées des bovins, des caprins et des ovins. Reprod. Nutr. Dév. 26, 279-280

Elhag C.A. (1976) A comparative study between desert goat and sheep efficiency of feed utilization. World Rev. Anim. Prod. 12, 43-48

Focant M. (1984) Comportement alimentaire, rumination, fermentations réticulo-ruminales et acides gras volatils plasmatiques comparés chez la chèvre et le mouton : influence du régime. Reprod. Nutr. Dév. 24, 239-250

Focant M., Vanbelle M. \& Godfroid S. (1986) Activités alimentaires et motricité du rumen chez la chèvre et le mouton, pour deux régimes mixtes foin-orge. Reprod. Nutr. Dév. 26, $277-$ 278

Geoffroy F. (1974) Etude comparée du comportement alimentaire et mérycique de deux petits ruminants : la chèvre et le mouton. Ann. Zootech. 23, 63-73

Gihad E.A. (1876) Intake digestibility and nitrogen utilization of tropical natural grass hay by goats and sheep. J. Anim. Sci. 43, 878-883

Masson C., Alrahmoun W. \& Tisserand J.L. (1986) Etude comparée de la quantité ingérée, 
de la digestibilité, de l'utilization de l'azote, du temps moyen de rétention et du comportement alimentaire chez les jeunes caprins et ovins recevant différents régimes. Ann. Zootech. 35, 4960

Morand-Fehr P. (1981) Caractéristiques du comportement alimentaire et de la digestion des caprins. In : Symposium International "Nutrition et Systèmes d'Alimentation de La Chèvre", vol. 1 (Morand-Fehr P., Bourbouze A., De Simiane M., eds). ITOVIC, INRA, Tours, pp. 21-45 Ruckebusch Y. \& Bueno L. (1973) Un dispositif simple et autonome d'enregistrement de l'activité alimentaire chez les ovins au pâturage. Ann. Rech. Vet. 4, 627-636

Welch J.G. (1982) Rumination, particle size and passage from the rumen. J. Anim. Sci. 54, 885894 